\title{
Unconstrained Text Detection in Manga: a New Dataset and Baseline
}

\author{
Julián Del Gobbo ${ }^{1}$ and Rosana Matuk Herrera ${ }^{2}$ \\ ${ }^{1}$ Departamento de Computación, FCEN, Universidad de Buenos Aires, Argentina \\ 2 Departamento de Ciencias Básicas, Universidad Nacional de Luján, Argentina
}

\begin{abstract}
The detection and recognition of unconstrained text is an open problem in research. Text in comic books has unusual styles that raise many challenges for text detection. This work aims to binarize text in a comic genre with highly sophisticated text styles: Japanese manga. To overcome the lack of a manga dataset with text annotations at a pixel level, we create our own. To improve the evaluation and search of an optimal model, in addition to standard metrics in binarization, we implement other special metrics. Using these resources, we designed and evaluated a deep network model, outperforming current methods for text binarization in manga in most metrics.
\end{abstract}

\section{Introduction}

Manga is a type of Japanese comic book with a huge diversity of text and balloons styles in unconstrained positions (Fig. 1). Japanese is a highly complex language, with three different alphabets and thousands of text characters. It also has about 1200 different onomatopoeia, which frequently appear in manga. Furthermore, characters often look very similar to the art in which they are embedded. These complexities make a text detection method for manga challenging to design.

A translation process of a manga picture consists of detecting the text, erasing it, inpainting the image, and writing the translated text on the image. As it is an intricate process, the translation is usually done manually in manga, and only the most popular mangas are translated. The complexity of the Japanese language hinders the diffusion of manga, and other Japanese artworks, outside of Japan. Automating the translation would lead to solving the linguistic barrier. In this work, we focus on the first step of the translation process: text detection. To achieve high-quality inpainting of the image, the text detection should be made at a pixel level.

On one side, many works in text detection in comics have taken a balloon detection approach. However, in manga, the text and balloons are also part of the artwork. Thus, balloons could have a multiplicity of shapes and styles. Besides, the text can be outside the dialogue balloons (Figs. 1a|1c|d 1d), or inside the balloon there could be non-text contents (Fig. 1b), making a balloon detection approach unsuitable for this task. On the other side, most previous works in text detection have taken a box detection approach. However, manga contains 


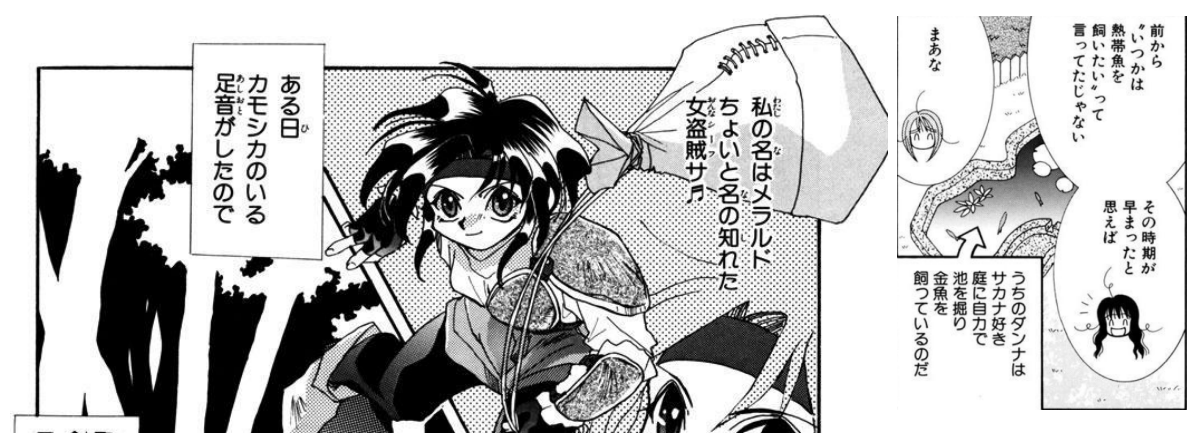

(b)

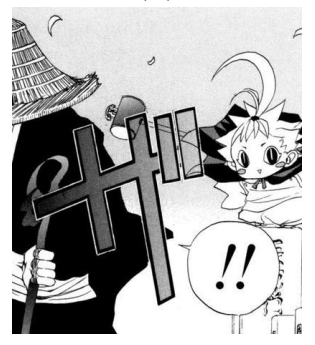

(c)

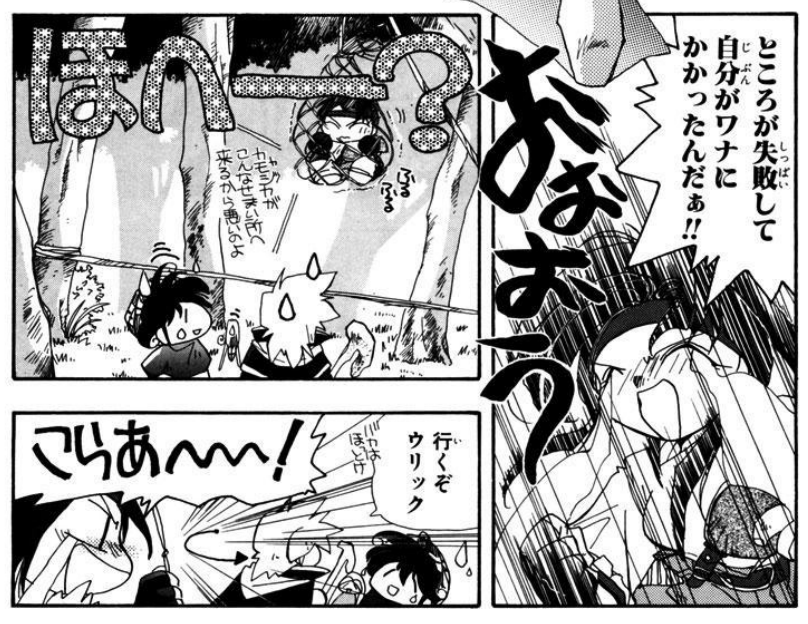

(a)

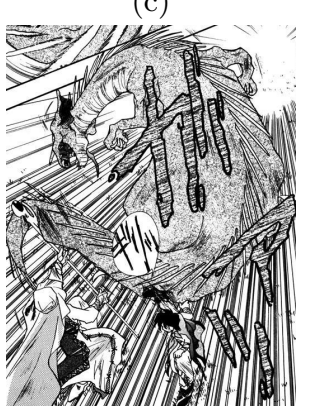

(d)

Fig. 1: Pictures showing the diversity of text styles in manga. (a) The dialogue balloons could have unconstrained shapes and border styles. The text could have any style and fill pattern, and could be written inside or outside the speech balloons. Note also that the frames could have non-rectangular shapes, and the same character could be in multiple frames. (b) Example of manga extract featuring non-text inside speech bubbles. (c) The same text character can have diverse levels of transparency. (d) Text characters could have a fill pattern similar to the background. All images were extracted from the Manga109 dataset [1] 2] [3]: (a) and (d) "Revery Earth" (c)Miyuki Yama, (b) "Everyday Oasakana-chan" (C) Kuniki Yuka, (c) "Akkera Kanjinchou" (C)Kobayashi Yuki

texts that are deformed, extremely large, or are drawn on the cartoon characters, which are hard to identify with a single bounding box (Figs. 1a $1 \mathrm{c}$ 1d). Thus, 
we decide to make text segmentation at a pixel level, identifying pixels as either text or background.

This work aims to detect unconstrained text characters in manga at a pixel level. The main contributions of this work are the following:

- The implementation and public release of many metrics for text detection at a pixel level, which others may reutilize, providing an easy way to find better models and compare results (Section 3).

- The creation and public release of a new dataset on text segmentation at a pixel level of Japanese manga (Section 4). To the best of our knowledge, this is the first dataset of these characteristics in manga.

- A simple and efficient model based on the U-net architecture [4 that outperforms state-of-the-art works on text detection in Japanese manga, with good baseline metrics for future works (Sections 5 and 66).

Despite the focus in manga, many techniques proposed in this work are general and could be used to do unconstrained text detection in other contexts.

\section{Related Work}

Speech balloon detection Several works have studied speech balloon detection in comics [56/8. While this could be used to detect speech balloons and then consider its insides as text, the problem is that text in manga is not always inside speech balloons. Furthermore, there are a few cases where not everything inside the balloon is text (Fig. 1b).

Bounding box detection Other works in text detection in manga, such as Ogawa et al. [3] and Yanagisawa et al. [9], have focused on text bounding box detection of multiple objects, including text. Wei-Ta Chu and Chih-Chi Yu have also worked on bounding box detection of text 10. Without restricting to manga or comics, there are many works every year that keep improving either bounding box or polygon text detection, one of the most recent ones being Wang et al. 11. However, methods trained with rigid word-level bounding boxes exhibit limitations in representing the text region for unconstrained texts. Recently, Baek et al.proposed a method (CRAFT) [12 to detect unconstrained text in scene images. By exploring each character and affinity between characters, they generate non-rigid word-level bounding boxes.

Pixel-level text segmentation There is a long history of text segmentation [13] and image binarization [14] 15] 16, 17] in the document analysis community related to historical manuscripts [18], maps [19], handwritten text [20], documents 21 and more. One of such works that does pixel level segmentation of text in document images is BCDU-net 22 . However, in these works most of the image is text along with a few lines or figures, and the text is more simple than one of manga, which features a lot more context, wide variety of shapes and styles. Outside the document binarization community, there are very few works that do pixel-level segmentation of characters, as there are few datasets available with pixel-level ground truth. One of such works is from Bonechi et al. [23]. 
As numerous datasets provide boundingbox level annotations for text detection, the authors obtained pixel-level text masks for scene images from the available boundingboxes exploiting a weakly supervised algorithm. However, a dataset with annotated bounding boxes should be provided, and the bounding box approach is not suitable for unconstrained text. Some few works that make pixel text segmentation in manga could be found on GitHub. One is called "Text Segmentation and Image Inpainting" by yu45020 24] and the other "SickZilMachine" by U-Ram Ko [25] 26]. Both attempt to generate a text mask in the first step via image segmentation and inpainting with such mask as a second step. In SickZil-Machine, the author created pixel-level text masks of the Manga109 dataset, but has not publicly released the labeled dataset. The author neither released the source code of the method but has provided an executable program to run it. In yu45020's work, the source code has been released.

Pixel-level segmentation Outside text, there are multiple works that do segmentation of different objects. One of such works is HRNet [27] [28, which has top scores in many tasks, including segmentation with cityscapes dataset [29].

\section{Evaluation Metrics}

Metrics such as recall, precision, and F-measure or $\mathrm{F}_{1}$ score 30] are widely used to evaluate binary segmentation models in images. These are defined as:

$$
\begin{gathered}
\text { Precision }=\frac{T P}{T P+F P} \\
\text { Recall }=\frac{T P}{T P+F N} \\
F-\text { measure }=F_{1}=\text { Pixel } F 1=P F 1=\frac{2 \text { Recall Precision }}{\text { Recall }+ \text { Precision }}
\end{gathered}
$$

with TP being pixels that were correctly segmented as text (true positive), FP being pixels that were wrongly segmented as text (false positives) and FN being pixels that were wrongly segmented as background (false negatives). Other standard evaluation measures in document binarization are PSNR and DRD 31. PSNR is a similarity measure between two images. The higher the value of PSNR, the higher the similarity of the two images. The Distance Reciprocal Distortion Metric (DRD) has been used to measure the visual distortion in binary document images [32. The lower the DRD, the lower the distortion.

Recall, precision and, F-measure metrics assume that the data is perfectly labeled and allow no compromises on the boundary, which is the part most prone to error. In many tasks, such as segmenting vehicles, this doesnt matter much as the area of a car is very big compared to the area that might be wrongly labeled, so the human error in labeling wont account much to influence metrics. 
With text, however, this is not the case. Not only are characters usually small, but also the boundary is many times unclear because of artifacts and blurring, as noted in Fig. 2, Another issue is that a large text character can have the same area as 100 small characters, making a model that correctly matches most of its pixels but none of the other 100 characters, as good as one matching the 100 small ones but little of the big one.

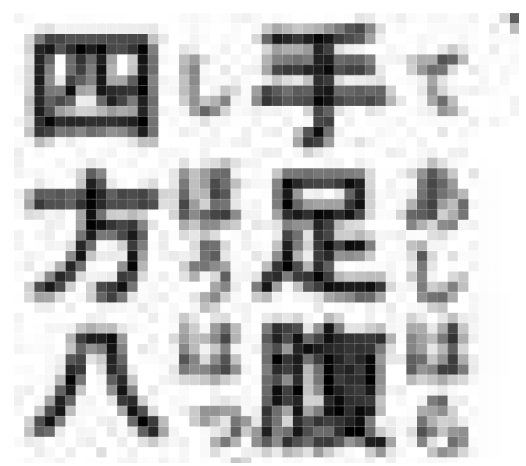

Fig. 2: Example of text inside the speech bubble zoomed in. Note that text boundary is unclear and prone to error due to artifacts. Image from "Akkera Kanjinchou" (C)Kobayashi Yuki, Manga109 dataset [1] 2] 3.

Calarasanu et al. 33 34 35 have proposed several metrics to account for these issues. In this work, we have adopted an approach similar to theirs. In addition to the standard pixel metrics, we calculate metrics based on connected components. A connected component in these images is a region of adjacent pixels, considering its 8 neighbors, sharing the same value (see Fig. 3a).

Given a ground truth connected component $G_{i}$ and its matching detection $D_{j}$, its accuracy and coverage are defined as:

$$
\begin{aligned}
\operatorname{Acc}_{i} & =\frac{\operatorname{Area}\left(G_{i} \bigcap D_{j}\right)}{\operatorname{Area}\left(D_{j}\right)} \\
\operatorname{Cov}_{i} & =\frac{\operatorname{Area}\left(G_{i} \bigcap D_{j}\right)}{\operatorname{Area}\left(G_{i}\right)}
\end{aligned}
$$

To account for multiple detections matching a single ground truth or a single detection matching multiple ground truths, we apply the watershed algorithm to match prediction pixels to a single ground truth, as seen in Fig. 3 .

We define $t p$ as the number of ground truth connected components that have at least one pixel of detection associated with it. We define $f p$ as the number of detected connected components which had no correspondence to any ground truth (see Fig. 3b). 


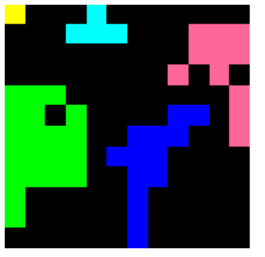

(a) Ground Truth

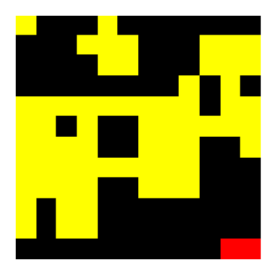

(b) Prediction

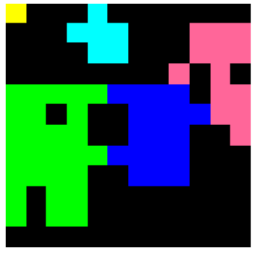

(c) Watershed

Fig. 3: Example of the watershed algorithm matching prediction pixels to ground truth connected components. (a) Masks of five ground truth connected components (labeled in different colors to be distinguished); (b) Predicted text segmentation mask. We marked in red (bottom, right) a predicted connected component with no correspondence to any ground truth connected component; (c) The predicted mask is matched with the ground truth using the watershed algorithm to obtain the evaluation metrics. Five detections matching ground truth connected components are obtained

Given a dataset with $m$ ground truth connected components and $d$ detections, we define the following metrics: quantity recall $R_{\text {quant }}$, quantity precision $P_{\text {quant }}$, quality recall $R_{\text {qual }}$, quality precision $P_{\text {qual }}$ and $\mathrm{F} 1$ quality $F 1_{\text {qual }}$ :

$$
\begin{array}{r}
R_{\text {quant }}=\frac{t p}{m} \\
P_{\text {quant }}=\frac{t p}{t p+f p} R_{\text {qual }}=\frac{\sum_{i=0}^{t p} C o v_{i}}{t p} \\
P_{\text {qual }}=\frac{\sum_{i=0}^{t p} A c c_{i}}{t p} \\
F 1_{\text {qual }}=\frac{2 R_{\text {qual }} P_{\text {qual }}}{R_{\text {qual }}+P_{\text {qual }}}
\end{array}
$$

Global recall $G R$, global precision $G P$, and global $\mathrm{F}_{1} G F 1$ are defined as:

$$
\begin{aligned}
G R=R_{\text {quant }} R_{\text {qual }} & =\frac{\sum_{i=0}^{t p} \operatorname{Cov}_{i}}{m} \\
G P=P_{\text {quant }} P_{\text {qual }} & =\frac{\sum_{i=0}^{t p} A c c_{i}}{t p+f p} \\
G F 1 & =\frac{2 G R G P}{G R+G P}
\end{aligned}
$$

We calculate metrics in normal and relaxed mode. Normal mode assumes that the dataset is perfectly labeled. Relaxed mode tries to lessen the effect of wrong boundary labeling (Fig. 4). In normal mode, we calculate the metrics using the segmentation masks of the dataset without modification. In relaxed 
mode, an eroded version of the ground truth is used to calculate coverage while a dilated version is used to calculate accuracy. In both modes, we consider there is no match to a ground truth component when there is no intersection between the eroded version and prediction, as the eroded version is the most relevant part to detect. For both erosion and dilation, a cross-shaped structuring element is used (connectivity=1).

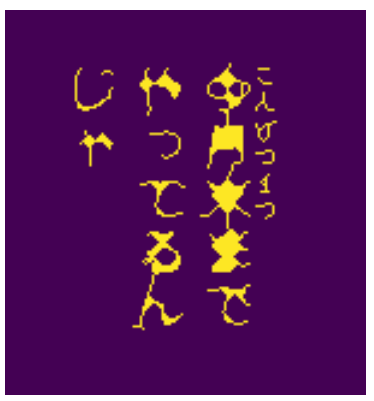

(a) Eroded GT

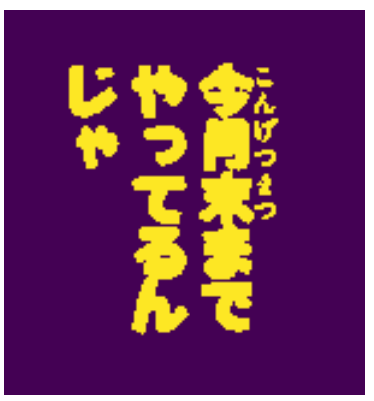

(b) Original GT

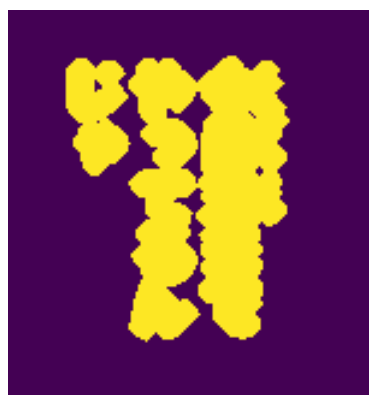

(c) Dilated GT

Fig. 4: Example of segmentation masks used in normal mode and relaxed mode metrics. (a) The eroded mask under-segments the ground truth mask. It is used for coverage in relaxed mode; (b) Ground truth mask. It is used in normal mode; (c) The dilated mask over-segments the ground truth. It is used for accuracy in relaxed mode. In relaxed mode, if the network predicts the ground truth of b), it has $100 \%$ of accuracy. However, if the prediction is inside the dilated mask of c), it also has $100 \%$ of accuracy. Accuracy is measured with Equation 4 .

\section{Dataset}

There are very few datasets of images with text and their corresponding pixel level mask (Table 1). This is mainly due to the large amount of time required to label them properly. However, most of them correspond to real-world images, which differ greatly from manga, and lack useful context such as speech balloons. Besides, most text in these datasets is in English and manga is in Japanese. Last, in these datasets, the text fonts are not hand-drawn in unrestricted orientations and with artistic styles like in manga.

Manga109 [1] 2] is the largest public manga dataset, providing bounding boxes for many types of objects, including text. However, it does not have pixel-level masks, and not all text has a bounding box. It is composed of 109 manga volumes drawn by professional manga artists in Japan, with all pages in black and white except for the first few pages of each volume. This is because unlike American and European comics which tend to be in color, manga is usually in black and white. 
Table 1: Datasets with pixel-level segmentation masks of text characters

\begin{tabular}{|c|c|c|c|c|c|}
\hline Dataset & Type & Language & Script & Images & Orientation \\
\hline $\begin{array}{c}\text { ICDAR 2013 Scene } \\
\text { Text Challenge [36] }\end{array}$ & Scene & English & Latin & 462 & Horizontal \\
\hline $\begin{array}{c}\text { ICDAR 2013 Born } \\
\text { Digital Challenge [36 }\end{array}$ & Digital & English & Latin & 551 & Horizontal \\
\hline $\begin{array}{c}\text { Total-Text } \\
\text { (2017) [37] }\end{array}$ & Scene & English & Latin & 1555 & $\begin{array}{c}\text { Multi-oriented, } \\
\text { Curve }\end{array}$ \\
\hline $\begin{array}{c}\text { DIBC0 [17] } \\
(2009-2019)\end{array}$ & Document & $\begin{array}{l}\text { European } \\
\text { languages }\end{array}$ & Latin & 136 & Horizontal \\
\hline KAIST[38] & Scene & $\begin{array}{c}\text { English, } \\
\text { Korean }\end{array}$ & Latin,Korean & 2483 & Horizontal \\
\hline Ours & Document & Japanese & $\begin{array}{l}\text { Kanji,Hiragana, } \\
\text { Katakana,Latin }\end{array}$ & 900 & Unrestricted \\
\hline
\end{tabular}

Datasets of synthetic images could be made using manga-style images, such as the images of the Danbooru2019 dataset 39, selecting the images without text, and adding text to them of a particular font and size. However, randomly adding text characters anywhere does not replicate where the text is naturally placed in manga, as much text is inside speech bubbles. Synthetically replicating the speech balloons is not easy either, as they are not always a simple rectangle or ellipse like shape. Besides, text outside speech balloons are part of the artwork, and usually have artistic styles of the author.

Taking into account all these issues, we decided to create our own dataset with pixel-level annotations. We chose to use images from Manga109, as it is a known public dataset, features a wide range of genres and styles, and the manga authors have granted permission to use and publish their works for academic research. To cover as many different styles as possible, few images from many manga volumes are preferable to a lot from few volumes, as long as those few are enough for the network to learn its style. After observing many examples, we concluded that the first ten images of each manga volume in the Manga109 dataset were a suitable number, as that included the cover of the manga and a few pages of the actual content. Thus we manually annotated with pixel-level text masks the first ten images from 45 different digital mangas, totalizing 450 images. As each manga image in the Manga109 dataset corresponds to 2 pages of a physical manga, we digitally annotated 900 physical pages of mangas.

Instead of a simple binary mask (text and non-text), we label the dataset with 3 classes (Fig. 5 . b): non-text, easy text (text inside speech balloons), and hard text (text outside speech balloons). While we still use the binary version for training, we use this separation of difficulties on text characters for a better understanding of model performance in metric evaluation.

In Table 2 we show standard metrics for a U-Net model trained on diverse datasets and tested with our dataset. It is clear that the segmentation of text in manga is greatly improved if the model is trained with our dataset. 


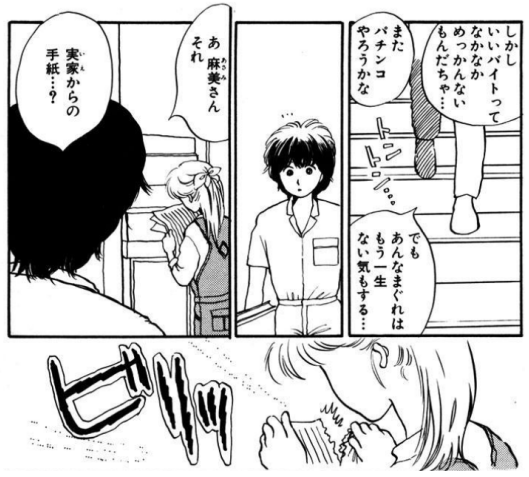

(a)

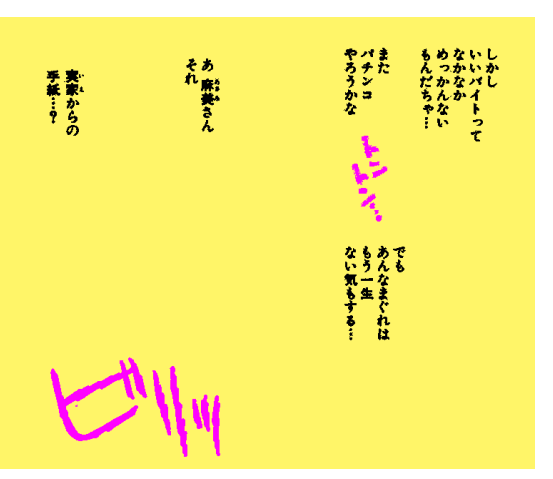

(b)

Fig. 5: Example of segmentation mask in our dataset. (a) Original image. Speech text is usually inside balloons and sound effects outside. Note the sound effects, near the stairs, and ripped paper. Image from "Aisazu Niha Irarenai" (CYoshi Masako, Manga109 dataset [1] 2] 3]. (b) Corresponding segmentation mask in our dataset. The text inside speech balloons is considered as an easy detection task and labeled with black. Text outside balloons is considered a difficult text detection and labeled with pink. Non-text pixels are labeled with yellow

Table 2: Metrics on our dataset training with other datasets. All trained with resnet34 U-Net for 10 epochs, saving model when PF1 score improved on manga dataset. To test against our own dataset, K-fold was used over 5 folds. GF1 is based on connected components while the rest are all pixel based metrics. Precision refers to Eq. 11 and recall to Eq. 2. Synthetic Text Danbooru2019 is a dataset that we built taking 75929 images without text from the Danbooru2019 [39] dataset and adding randomly Japanese characters from machine fonts.

\begin{tabular}{|c|c|c|c|c|c|c|}
\hline Dataset & PF1 & GF1 & Precision & Recall & DRD & PSNR \\
\hline DIBCO & 26.32 & 13.47 & 19.09 & 42.37 & 74.71 & 11.64 \\
ICDAR 2013 Scene Text Images & 45.86 & 40.47 & 39.37 & 54.91 & 38.14 & 14.53 \\
ICDAR 2013 Born-Digital Images & 40.90 & 39.67 & 51.94 & 33.73 & 28.42 & 16.22 \\
Total-Text & 42.49 & 42.79 & 47.10 & 38.70 & 29.19 & 15.66 \\
KAIST & 45.69 & 29.17 & 44.55 & 46.90 & 32.64 & 15.30 \\
Synthetic Text Danbooru2019 & 57.03 & 42.83 & 76.01 & 45.64 & 18.88 & 18.24 \\
Ours & $\mathbf{7 6 . 2 2}$ & $\mathbf{8 2 . 6 4}$ & $\mathbf{8 0 . 4 1}$ & $\mathbf{7 2 . 5 0}$ & $\mathbf{1 2 . 8 3}$ & $\mathbf{2 1 . 0 6}$ \\
\hline
\end{tabular}

\section{Methodology}

Our text detector model employs a U-net [4 architecture with a pre-trained resnet34 [40] backbone. Despite having been pre-trained with ImageNet, which features images quite different from manga, it has proved to work well. We implemented the model in PyTorch. We used the fastai U-Net model [41] and trained the network with the fastai library [42] 43, making use of its one cycle 
policy, a modified version of the one initially devised by Leslie N. Smith 44. The encoder part was frozen, and only the decoder part was trained, as the encoder already comes with the pre-trained weights from ImageNet. As we handle binary segmentation, a single channel is used as the last layer to provide the logits of a pixel being text. We later apply a sigmoid function and set 0.5 as a threshold to consider whether to classify it as text or background. As for the loss function, dice loss is used, which showed considerably better results than the simple binary cross-entropy loss.

The images of the Manga109 dataset are 1654 width and 1170 height. As they represent sheets of paper from physical books, in almost all cases (with some covers as the exception), the two pages from it have no text in the middle and can be split without affecting text characters. We took advantage of that and cut the images of our dataset in half, so we end up with 900 manga pages to train (see Section 4). The only data augmentation used is a $512 \times 800$ random crop for training. We tried a few other data augmentations such as flip and warp, but we didnt notice any significant improvement.

We used K-Fold cross-validation with five folds to calculate all metrics, leaving $20 \%$ as validation. Between transfer learning, one cycle policy, and a batch size of 4 , results are obtained by training for ten epochs, which is completed in less than an hour on a single GeForce GTX 1080 Ti GPU for a single fold.

In the next section, we show how we used our metrics of Section 3 to select an optimal loss function and an optimal architecture for the model.

\section{Experiments}

\subsection{Loss Function Selection}

Choosing an adequate loss function is a crucial step in the design of a machine learning model. We used our dataset to train a U-net network, which is a model commonly used in segmentation, with different loss functions. Then, we used the metrics of section 3 to measure the performance of the model for each different loss function.

We trained a fastai resnet18 U-net with each loss function during ten epochs, with 0.001 as the maximum learning rate. After trying different loss functions such as boundary loss, binary cross entropy and focal loss the highest scores in the metrics were obtained by $-\log$ (DiceLoss) [45]. Thus, this was the loss function that we choose for further experiments.

\subsection{Model Performance}

To create a good baseline, we made experiments with different methods and use the metrics to compare their performance for the binarization task of our dataset. The experimental results are shown in Table 3.

The yu45020's xception [24, the yu45020's mobileNetV2 [24] and the "SickZilMachine" 25] 26] are methods we found on Github that also aim to do pixel-level 
text segmentation in manga. We trained each one of the yu45020's architectures with our dataset for 10 epochs with all layers unfrozen. For SickZil-Machine the training code is not available so we used their executable to generate the predictions of Table 3 .

Table 3: Performance metrics obtained by similar methods under similar conditions for the task of binarizing our manga dataset. All methods were trained with our Manga109 labeled dataset except SickZil-Machine because the author did not release the source code. The results shown for SickZil-Machine are for an executable program provided by the author. However, SickZil-Machine was trained by his author with its own Manga109 dataset in which text was labeled at a pixel level. Precision refers to Eq. 1 and recall to Eq. 2 .

\begin{tabular}{|c|c|c|c|c|c|c|c|c|}
\hline \multirow{2}{*}{ Author } & \multicolumn{4}{|c|}{ Normal } & \multicolumn{2}{c|}{ Relaxed } \\
\cline { 2 - 9 } & PF1 & GF1 & Precision & Recall & DRD & PSNR & PF1 & GF1 \\
\hline BCDU-net [22] & 30.24 & 15.08 & 40.54 & 27.32 & 39.42 & 14.80 & 27.38 & 16.19 \\
yu45020's xception & 61.61 & 62.48 & 61.25 & 62.09 & 20.66 & 17.77 & 67.48 & 79.35 \\
yu45020's mobileNetV2 & 47.97 & 39.17 & 49.43 & 46.88 & 28.12 & 16.12 & 50.54 & 52.26 \\
SickZil-Machine & 52.07 & 49.33 & 41.77 & 69.15 & 35.75 & 14.71 & 64.66 & 84.94 \\
HRNet [27] [28] & 51.64 & 51.34 & 43.40 & 63.85 & 33.27 & 15.40 & 63.36 & 74.41 \\
fastai Resnet18 U-Net & 75.82 & 83.29 & 75.53 & 76.32 & 14.06 & 21.35 & 76.87 & 87.50 \\
fastai Resnet34 U-Net & $\mathbf{7 9 . 3 6}$ & $\mathbf{8 4 . 9 2}$ & $\mathbf{8 2 . 2 6}$ & $\mathbf{7 6 . 7 1}$ & $\mathbf{1 1 . 1 5}$ & $\mathbf{2 1 . 9 1}$ & $\mathbf{8 0 . 4 3}$ & $\mathbf{8 9 . 2 6}$ \\
\hline
\end{tabular}

We also compared against BCDU-net [22, a recent method for segmentation that has shown good results on multiple DIBCO datasets [46. BCDU-net is a deep auto-encoder-decoder network. This method applies bidirectional convolutional LSTM layers in U-net structure to non-linearly encode both semantic and high-resolution information with non-linearly technique. We chose the BCDU-net implementation that was based on DIBCO 46] and trained it with our dataset in 5 folds, generating 40 random 128x128 patches from each image. Each fold was trained for 20 epochs.

HRNet [27] 28] is a very promising method for visual recognition with stateof-the-art results in many tasks and datasets. The authors show the superiority of the HRNet in a wide range of applications, including human pose estimation, semantic segmentation, and object detection. The configuration that we used was similar to the one provided for the cityscapes segmentation [47. We trained each fold for 100 epochs each, changing the loss to -log(DiceLoss).

We also experimented with a lot of variations of the U-Net architecture provided with fastai library. As shown in Table 3. the best binarization results were achieved by a fastai Resnet34 U-Net. For this network, we trained the decoder for 10 epochs, further trained the whole model for 5 epochs, and finally trained with whole images instead of random crops for 3 additional epochs. We kept this network as our baseline model because it achieved the top metric scores.

Last, we also experimented with 23 segmentation models from qubvel's library [4], training only the decoder and using the default parameters. However, 
the fastai U-Net network outperformed the models in this library in all metrics for this problem.

Our metrics allow us to find a simple and efficient model, that outperforms the other models especially on normal mode, as shown in Table 3.

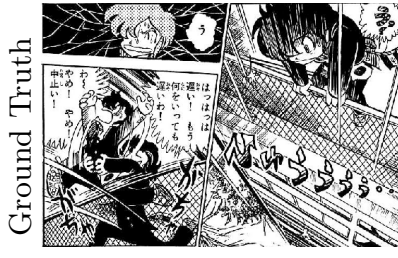

(a) Manga image

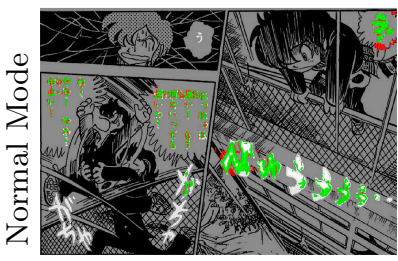

(d) yu45020's xception

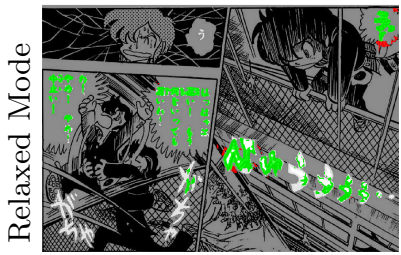

(g) yu45020's xception

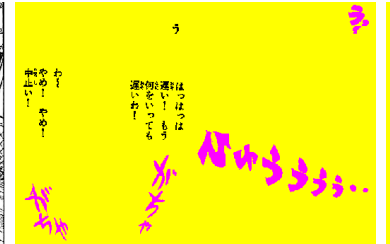

(b) Ground Truth

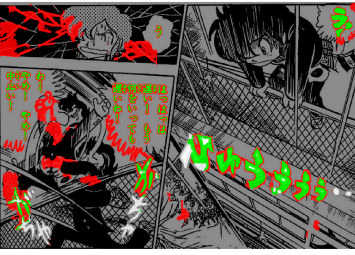

(e) SickZil-Machine

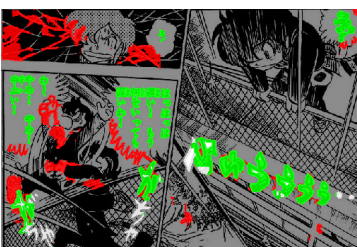

(h) SickZil-Machine

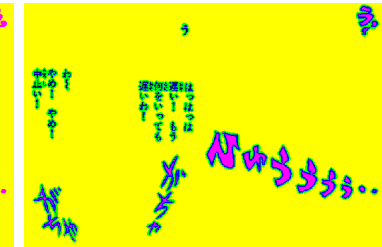

(c) Relaxed GT

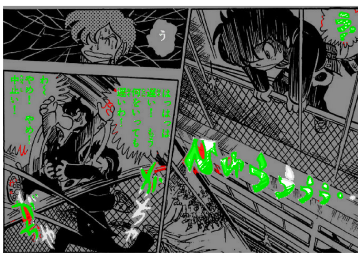

(f) Ours

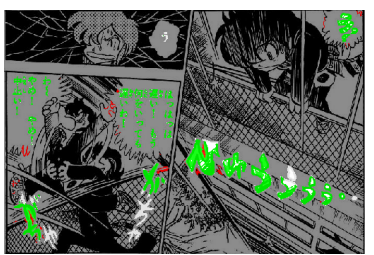

(i) Ours

Fig. 6: Segmentation masks obtained by different methods. In red, false positives. In white, missing text. In green, text correctly segmented. (a) Image extracted from "BEMADER_P" (C)Hasegawa Yuichi, Manga109 dataset 1] 2] 3]. (b) Ground Truth. In pink, hard text. In black, easy text. (c) Relaxed Ground Truth. In green, dilated area. In blue, ground truth pixels, that does not belong to the eroded mask. (d, e, f) Normal mode results. (g, h, i) Relaxed mode results. Note that in (h) boundaries between the small letters are lost, but they are still marked as true positives because the pixels are inside the relaxed dilated area

Fig. 6 shows an example of segmentation masks produced by the different methods. Our segmentation method misses some of the hard texts but has very few false positives (Figs. 6f 6ii). SickZil-Machine covers some of those missing texts but also has much more false positives (Figs. 6e, 6h). yu45020s xception misses many of the hard texts, and detects the small letters with less precision than our model (Figs. 6d, 6g). 
For a global view of the performance on the different types of connected components and segmentation modes, we draw in Fig. 7 the histograms of $F 1_{\text {qual }}$ (see Equation 9. As our method fits the text characters without over-segmentation, it has less false positives, and our method clearly outperforms the other methods for $F 1_{\text {qual }}$ in normal mode. For the easy text case in relaxed mode, our method and SickZil-Machine detect almost all the connected components. Thus, we can see that there is little point in adding more data of easy text, as almost all easy components are detected with high $\mathrm{F}_{1}$ score.

\section{Conclusions}

The detection and recognition of unconstrained text is an open problem in research. Standard methods developed for the Latin alphabet do not perform well with Japanese, due to Japanese having many more characters: about 2,800 common characters out of a total set of more than 50,000. Besides, each Japanese character is, on average, more complicated than an English letter. Japan is a country with an immense cultural heritage. Unfortunately, the complexity of the Japanese language constitutes a linguistic barrier for accessing its culture. Automatic translation methods would contribute to overcome it.

In this work, we presented a study into unconstrained text segmentation at a pixel level in Japanese manga. We created a dataset manually annotating the text of manga images at a pixel level. Besides, we implemented special and standard metrics to evaluate the binarization task. We show that these tools, together with the fastai library, allowed us to find a simple and efficient deep learning model that outperforms in most metrics previous works on the same task. Despite our focus in manga, the techniques proposed in this work could be expanded to do unconstrained Japanese text detection in other contexts. For instance, the text segmentation masks obtained by our method could be useful for Japanese OCR and inpainting in other Japanese graphical documents.

The release of the dataset and metrics provided by this work would also enable other researchers and practitioners to find better models for this problem and compare results. To the best of our knowledge, this would be the first public dataset on text segmentation at a pixel level of Japanese manga. 

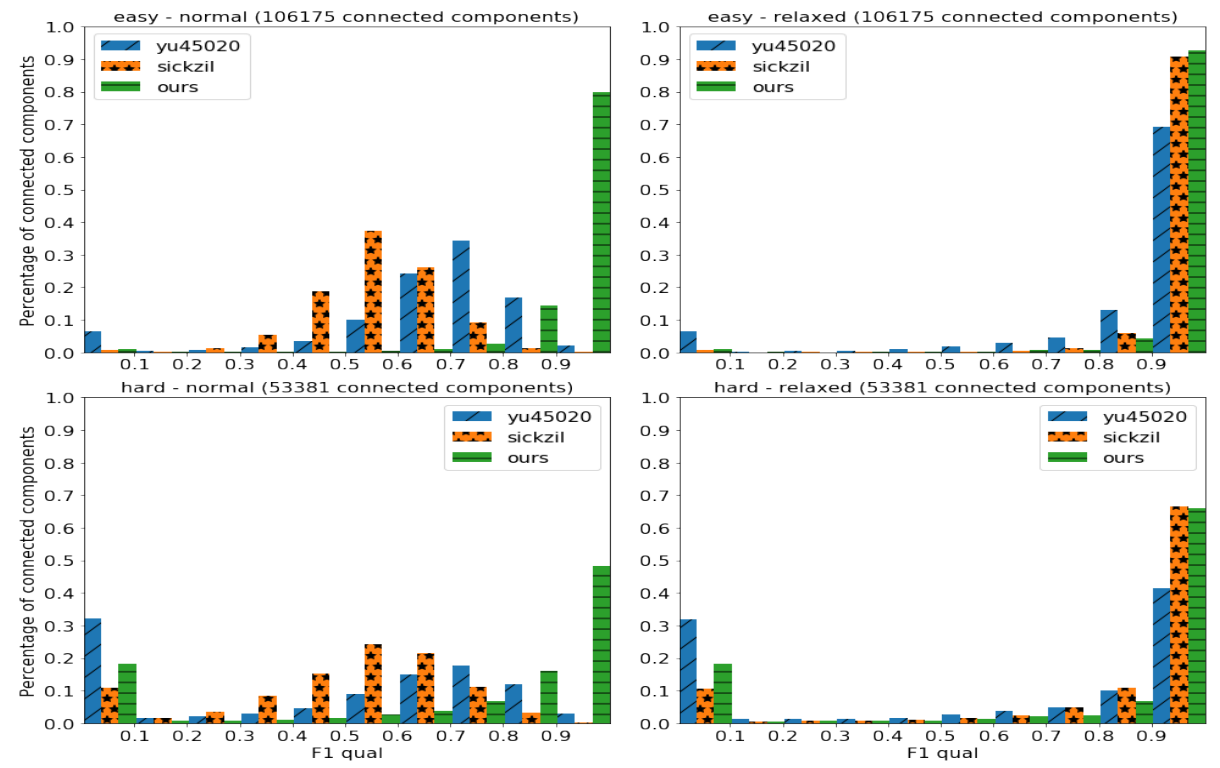

Fig. 7: Histogram of $\mathrm{F} 1_{\text {qual }}$ (see Eq. 9 of the different types of connected components. The first row corresponds with easy text, and the second row corresponds with hard text. The first column corresponds with normal mode, and the second column corresponds with relaxed mode. For easy text, our method predicts most of the connected components with a high $F 1_{\text {qual }}$ value. In normal mode our method has a much higher percentage of easy and hard connected components predicted with a high $F 1_{\text {qual }}$ value than the other methods.

\section{Code}

https://github.com/juvian/Manga-Text-Segmentation 


\section{References}

1. Manga109: Japanese manga dataset. http://www.manga109.org/en/

2. Matsui, Y., Ito, K., Aramaki, Y., Fujimoto, A., Ogawa, T., Yamasaki, T., Aizawa, K.: Sketch-based manga retrieval using manga109 dataset. Multimedia Tools and Applications 76(20) (2016) 2181121838

3. Ogawa, T., Otsubo, A., Narita, R., Matsui, Y., Yamasaki, T., Aizawa, K.: Object detection for comics using manga109 annotations. ArXiv abs/1803.08670 (2018)

4. Ronneberger, O., Fischer, P., Brox, T.: U-net: Convolutional networks for biomedical image segmentation. In: Medical Image Computing and Computer-Assisted Intervention (MICCAI). Volume 9351 of Lecture Notes in Computer Science. Springer (2015) 234-241

5. Rigaud, C., Burie, J.C., Ogier, J.M.: Text-independent speech balloon segmentation for comics and manga. In: GREC. (2015)

6. Nguyen, N., Rigaud, C., Burie, J.: Comic MTL: optimized multi-task learning for comic book image analysis. International Journal on Document Analysis and Recognition (IJDAR) 22(3) (2019) $265-284$

7. Liu, X., Li, C., Zhu, H., Wong, T.T., Xu, X.: Text-aware balloon extraction from manga. The Visual Computer 32(4) (2016) 501511

8. Dubray, D., Laubrock, J.: Deep CNN-based speech balloon detection and segmentation for comic books. 2019 International Conference on Document Analysis and Recognition (ICDAR) (2019) 1237-1243

9. Yanagisawa, H., Yamashita, T., Watanabe, H.: A study on object detection method from manga images using CNN. 2018 International Workshop on Advanced Image Technology (IWAIT) (2018) 1-4

10. Chu, W.T., Yu, C.C.: Text detection in manga by deep region proposal, classification, and regression. 2018 IEEE Visual Communications and Image Processing (VCIP) (2018) 1-4

11. Wang, W., Xie, E., Li, X., Hou, W., Lu, T., Yu, G., Shao, S.: Shape robust text detection with progressive scale expansion network. In: Proceedings of the IEEE Conference on Computer Vision and Pattern Recognition. (2019) 9336-9345

12. Baek, Y., Lee, B., Han, D., Yun, S., Lee, H.: Character region awareness for text detection. In: Proceedings of the IEEE Conference on Computer Vision and Pattern Recognition. (2019) 9365-9374

13. Mehul, G., Ankita, P., Udeshi Namrata, D., Rahul, G., Sheth, S.: Text-based image segmentation methodology. Procedia Technology 14 (2014) 465-472

14. Fletcher, L.A., Kasturi, R.: A robust algorithm for text string separation from mixed text/graphics images. IEEE Trans. Pattern Anal. Mach. Intell. 10 (1988) 910-918

15. Wu, V., Manmatha, R., Riseman, E.M.: Finding text in images. In: Proceedings of the Second ACM International Conference on Digital Libraries. DL '97, New York, NY, USA, Association for Computing Machinery (1997) 312

16. Gatos, B., Ntirogiannis, K., Pratikakis, I.: ICDAR 2009 Document Image Binarization Contest (DIBCO 2009). In: 10th International Conference on Document Analysis and Recognition. (2009) 1375-1382

17. Pratikakis, I., Zagoris, K., Karagiannis, X., Tsochatzidis, L., Mondal, T., MarthotSantaniello, I.: ICDAR 2019 Competition on Document Image Binarization (DIBCO 2019). (2019) 1547-1556

18. Ntogas, N., Veintzas, D.: A binarization algorithm for historical manuscripts. In: ICC 2008. (2008) 41-51 
19. Biswas, S., Mandal, S., Das, A.K., Chanda, B.: Land map images binarization based on distance transform and adaptive threshold. In: 11th IAPR International Workshop on Document Analysis Systems. (2014) 334-338

20. Solihin, Y., Leedham, G.: Integral ratio: A new class of global thresholding techniques for handwriting images. IEEE Trans. Pattern Anal. Mach. Intell. 21 (1999) $761-768$

21. Mustafa, W., Kader, M.: Binarization of document images: A comprehensive review. Journal of Physics: Conference Series 1019 (2018) 012023

22. Azad, R., Asadi-Aghbolaghi, M., Fathy, M., Escalera, S.: Bi-directional convLSTM U-Net with densley connected convolutions (2019)

23. Bonechi, S., Andreini, P., Bianchini, M., Scarselli, F.: COCO_TS dataset: Pixellevel annotations based on weak supervision for scene text segmentation. In: ICANN. (2019)

24. yu45020: Text segmentation and image inpainting.https://github.com/yu45020/ Text_Segmentation_Image_Inpainting (2019)

25. Ko, U.R.: Sickzil-machine. https://github.com/KUR-creative/ SickZil-Machine (2019)

26. Ko, U.R., Cho, H.G.: Sickzil-machine: A deep learning based script text isolation system for comics translation. In Bai, X., Karatzas, D., Lopresti, D., eds.: Document Analysis Systems, Springer International Publishing (2020) 413-425

27. Sun, K., Xiao, B., Liu, D., Wang, J.: Deep high-resolution representation learning for human pose estimation. In: CVPR. (2019)

28. Wang, J., Sun, K., Cheng, T., Jiang, B., Deng, C., Zhao, Y., Liu, D., Mu, Y., Tan, M., Wang, X., Liu, W., Xiao, B.: Deep high-resolution representation learning for visual recognition. TPAMI (2019)

29. Cordts, M., Omran, M., Ramos, S., Rehfeld, T., Enzweiler, M., Benenson, R., Franke, U., Roth, S., Schiele, B.: The cityscapes dataset for semantic urban scene understanding. In: Proc. of the IEEE Conference on Computer Vision and Pattern Recognition (CVPR). (2016)

30. Chinchor, N.: Muc-4 evaluation metrics. In: Proceedings of the 4th Conference on Message Understanding. MUC4 '92, USA, Association for Computational Linguistics (1992) 2229

31. Pratikakis, I., Zagoris, K., Karagiannis, X., Tsochatzidis, L., Mondal, T., MarthotSantaniello, I.: ICDAR 2019 Competition on Document Image Binarization (DIBCO 2019). 2019 International Conference on Document Analysis and Recognition (ICDAR) (2019) 1547-1556

32. Lu, H., Kot, A., Shi, Y.: Distance-reciprocal distortion measure for binary document images. IEEE Signal Processing Letters 11 (2004) 228-231

33. Calarasanu, S.: Improvement of a text detection chain and the proposition of a new evaluation protocol for text detection algorithms. PhD thesis, Université Pierre et Marie Curie - Paris 6, Paris, France (2015)

34. Calarasanu, S., Fabrizio, J., Dubuisson, S.: From text detection to text segmentation: A unified evaluation scheme. In: Proceedings of the 2nd International Workshop on Robust Reading Conference (IWRR-ECCV). (2016)

35. Calarasanu, S., Fabrizio, J., Dubuisson, S.: What is a good evaluation protocol for text localization systems? Concerns, arguments, comparisons and solutions. Image Vis. Comput. 46 (2016) 1-17

36. Karatzas, D., Shafait, F., Uchida, S., Iwamura, M., Gomez i Bigorda, L., Mestre, S.R., Mas, J., Mota, D.F., Almazàn, J.A., de las Heras, L.P.: ICDAR 2013 Robust Reading Competition. In: Proc. 12th International Conference on Document Analysis and Recognition. ICDAR 13, IEEE Computer Society (2013) 14841493 
37. Chng, C.K., Chan, C.S., Liu, C.: Total-text: Towards orientation robustness in scene text detection. International Journal on Document Analysis and Recognition (IJDAR) $\mathbf{2 3}$ (2020) 31-52

38. Jung, J.H., Lee, S.H., Cho, M.S., Kim, J.H.: Touch TT: Scene text extractor using touchscreen interface. ETRI Journal 33 (2011) 78-88

39. Anonymous, community, D., Branwen, G.: Danbooru2019: A large-scale crowdsourced and tagged anime illustration dataset. https://www.gwern.net/ Danbooru2019 (January 2020)

40. He, K., Zhang, X., Ren, S., Sun, J.: Deep residual learning for image recognition. 2016 IEEE Conference on Computer Vision and Pattern Recognition (CVPR) (2015) 770-778

41. Fastai: U-net model. https://docs.fast.ai/vision.models.unet.html Accessed: 2020-03-02

42. Fastai: Fastai deep learning library. https://github.com/fastai/fastai Accessed: 2020-02-10.

43. Howard, J., Gugger, S.: fastai: A layered API for deep learning (2020)

44. Smith, L.N.: A disciplined approach to neural network hyper-parameters: Part 1 learning rate, batch size, momentum, and weight decay. ArXiv abs/1803.09820 (2018)

45. Milletari, F., Navab, N., Ahmadi, S.: V-net: Fully convolutional neural networks for volumetric medical image segmentation. In: 2016 Fourth International Conference on 3D Vision (3DV). (2016) 565-571

46. Azad, R., Asadi-Aghbolaghi, M., Fathy, M., Escalera, S.: BCDUnet DIBCO. https://github.com/rezazad68/BCDUnet_DIBC0 (2019)

47. Wang, J., Sun, K., Cheng, T., Jiang, B., Deng, C., Zhao, Y., Liu, D., Mu, Y., Tan, M., Wang, X., Liu, W., Xiao, B.: High-resolution networks (HRNets) for Semantic Segmentation. https://github.com/HRNet/HRNet-Semantic-Segmentation

48. qubvel: Segmentation models. https://github.com/qubvel/segmentation_ models . pytorch (2019) 